\title{
Assessment of morphological differences of the proximal tibia in healthy knees: analysis of the 3-dimensional mathematical model
}

\author{
Jolanta Tomczyk $^{1 \wedge}$, Joanna Taczała ${ }^{2}$, Jacek Sawicki ${ }^{2} \wedge$, Marcin Domżalski $^{3} \wedge$ \\ ${ }^{1}$ Department of Radiology, Isotopic Diagnostic and Therapy, Veteran's Memorial Hospital Medical University of Lodz, Lodz, Poland; ${ }^{2}$ Institute \\ of Materials Science and Engineering, Lodz University of Technology, Lodz, Poland; ${ }^{3}$ Department of Orthopedics and Traumatology. Veteran's \\ Memorial Hospital Medical University of Lodz, Lodz, Poland
}

Contributions: (I) Conception and design: All authors; (II) Administrative support: All authors; (III) Provision of study materials or patients: J Tomczyk, J Taczała; (IV) Collection and assembly of data: J Tomczyk, J Taczała; (V) Data analysis and interpretation: All authors; (VI) Manuscript writing: All authors; (VII) Final approval of manuscript: All authors.

Correspondence to: Jolanta Tomczyk. Department of Radiology, Isotopic Diagnostic and Therapy, Veteran's Memorial Hospital Medical University of Lodz, Poland Zeromskiego 113 Street, 90-549 Lodz, Poland. Email: jol.tomczyk@gmail.com.

Background: High tibial osteotomy and many orthopedic surgical procedures around the knee joint requires precise preoperative planning. In-depth knowledge of the tibial plateau morphology is necessary to limit intraoperative complications like lateral hinge fracture. No studies were exploring the differences in proximal tibia surface geometry, in regards to gender and laterality, using a mathematical model. The aim of our study was to assess morphological differences in healthy knees using a three-dimensional mathematical model.

Methods: Eighty-seven computed tomography examinations collected from 52 patients were selected for the study. The inclusion criteria were: age between 20 and 40 years, knee joint without visible deformities, no history of significant trauma to index knee, no history of systemic and chronic disorders. The average age of the included patients was $32.5 \pm 8.9$ years old. For the calculation and comparisons, 45 right knee joints (18 females and 27 males) and 42 left knee joints (17 females and 25 males) were used.

Results: The male tibial plateau was much larger than the female one, for the right $(\mathrm{P}=0.001)$ and left knees $(\mathrm{P}=0.001)$. Male knees showed much bigger variability in two-dimensional tibial plateau dimensions especially for the left knees $(\mathrm{P}=0.001)$, and there was also a marked difference in variability between sides in males. Three-dimensional variability was significant for medial condyles for both genders. Male knees had a statistically bigger $(\mathrm{P}=0.04)$ tibial plateau surface area for all measured condyles.

Conclusions: The proximal tibial plateau showed in the designed mathematical models high variability in the two-dimensional and three-dimensional analysis. The males' knees presented great variability between sides and condyles. This finding must be considered during preoperative planning.

Keywords: Tibia; three-dimensional model (3D model); anatomical variations

Submitted Sep 25, 2020. Accepted for publication Mar 26, 2021.

doi: 10.21037 /qims-20-1094

View this article at: https://dx.doi.org/10.21037/qims-20-1094

^ ORCID: Jolanta Tomczyk, 0000-0002-8942-0233; Joanna Taczała, 0000-0002-3201-9182; Jacek Sawicki, 0000-0001-9147-7338; Marcin Domżalski, 0000-0003-1915-0773. 


\section{Introduction}

There has been a long-standing interest in the geometric bilateral symmetry of the bones forming the knee joint (1-3). This interest is partly due to the symmetry postulation that is frequently made in clinical assessments and research studies. A common clinical practice is to use the contralateral unaffected side as a template for planning corrective osteotomy $(4,5)$.

Many templates designed for surgical planning of the procedures around the knee are using the contralateral limb as a reference, considering that the left and right extremities are not significantly different. Moreover, these templates assume that there are insignificant differences between males' and females' proximal tibia morphology.

Little is known, how chronic, degenerative diseases may alter the geometry of the articular surfaces of the knee joint. Osteoarthritis (OA) of the knee is often a cause of considerable deformation of the proximal tibia and previous studies have shown that this joint is more commonly affected by degenerative changes that any other in the human motor system (6).

Since the first Jackson report (7) in 1958 high tibial osteotomy (HTO) has been recognized for its value for the treatment of symptomatic arthritis of the knee, especially in a younger, more active patient (6). The success of this procedure is based on detailed preoperative planning of axial deformity of the lower extremity and congruency of the knee joint. HTO is predominantly scheduled for patients with unicompartmental medial OA, and varus knees. Since HTO changes the slope of the tibial plateau a better insight into the anatomical variations of the tibia plateau between genders and sides is mandatory for proper surgical planning. Lack of adequate, anatomical—based preparation for HTO may result in an intra-operative lateral hinge or intraarticular fracture and treatment failure (8).

The precise model of the proximal tibial epiphysis is required for more morphological and biomechanical studies.

The first aim of this study was to design a mathematical model for $3 \mathrm{D}$ reconstruction of average proximal tibia separately for males and females, as well as for right and left limbs. The second purpose was to find differences between created models concerning gender and dominant and nondominant limbs.

\section{Methods}

\section{Data collection}

Computed tomography (CT) scans of patients who had undergone CT scanning between 2015 to 2019 due to unrelated orthopedic reasons to the index knee joint (i.e., vascular indications, injuries to the opposite leg) were collected from the university hospital database. Becuase our study did not disclose any private petient data, individual consent for this retrospective analysis was waived. The inclusion criteria were: age between 20 and 40 years, knee joint without visible deformations, no history of significant trauma to index knee, no history of systemic and chronic disorders. Each CT scan was evaluated by a trained medical doctor for signs of radiological OA in the knee joint (such as joint space narrowing, subchondral sclerosis, subchondral cyst formation and osteophytes). Any CT scan exhibiting signs of OA was excluded from the study. All inclusion criteria fulfilled 87 CT examinations collected from 52 patients out of 454 individuals screened for inclusion/exclusion criteria. The study was conducted in accordance with the Declaration of Helsinki (as revised in 2013). An approval from the Local University Ethical Committee was obtained for the study design and data acquisition from CT scans. Because our study did not disclose any private patient data, individual consent for this retrospective analysis was waived.

The average age of the included patients was $32.5 \pm 8.9$ years old. There were 33 men and 19 women in the study group. For the calculation and comparisons, 18 females and 27 males right knee joints, and 17 females and 25 males left knee joints, were used.

\section{Imaging}

The CT scans were made using a Siemens SOMATOM Sensation 64 CT scanner. The acquisition parameters were the following: tube voltage $120 \mathrm{kVp}$, with automatic selection of exposure conditions-CARE DOSE, slice thickness $1 \mathrm{~mm}$, a matrix size of $512 \times 512$ pixels, and a pixel size of $1.52344 \mathrm{~mm}$, rotation time $1 \mathrm{~s}$, Pitch 0,55 .

The first part of the work was transforming 2D CT images into $3 \mathrm{D}$ geometry by the image segmentation process. This action was intended to obtain a virtual $3 \mathrm{D}$ model named later as CAD body (CAD-computer-aided design). Later, the models were edited in $3 \mathrm{D}$ modeling software to obtain a closed model needed for numerical calculations.

Then with GOM Inspect V8 SR1 (Braunschweig, Germany) and ANSYS 2019 R3 software (by ANSYS Inc. company), 3D models were analyzed. Right and left tibial bones as well as the medial and lateral tibial condyle plateau were analyzed separately. 


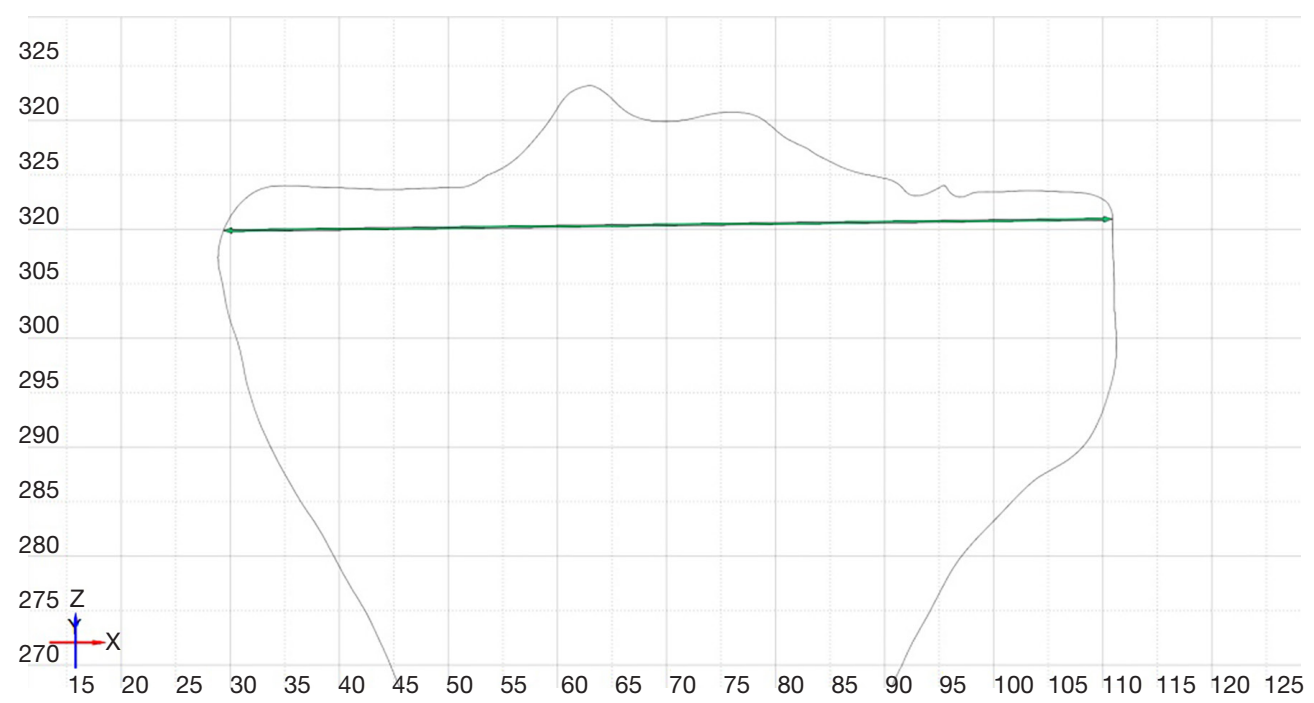

Figure 1 Exemplary place of measurement.

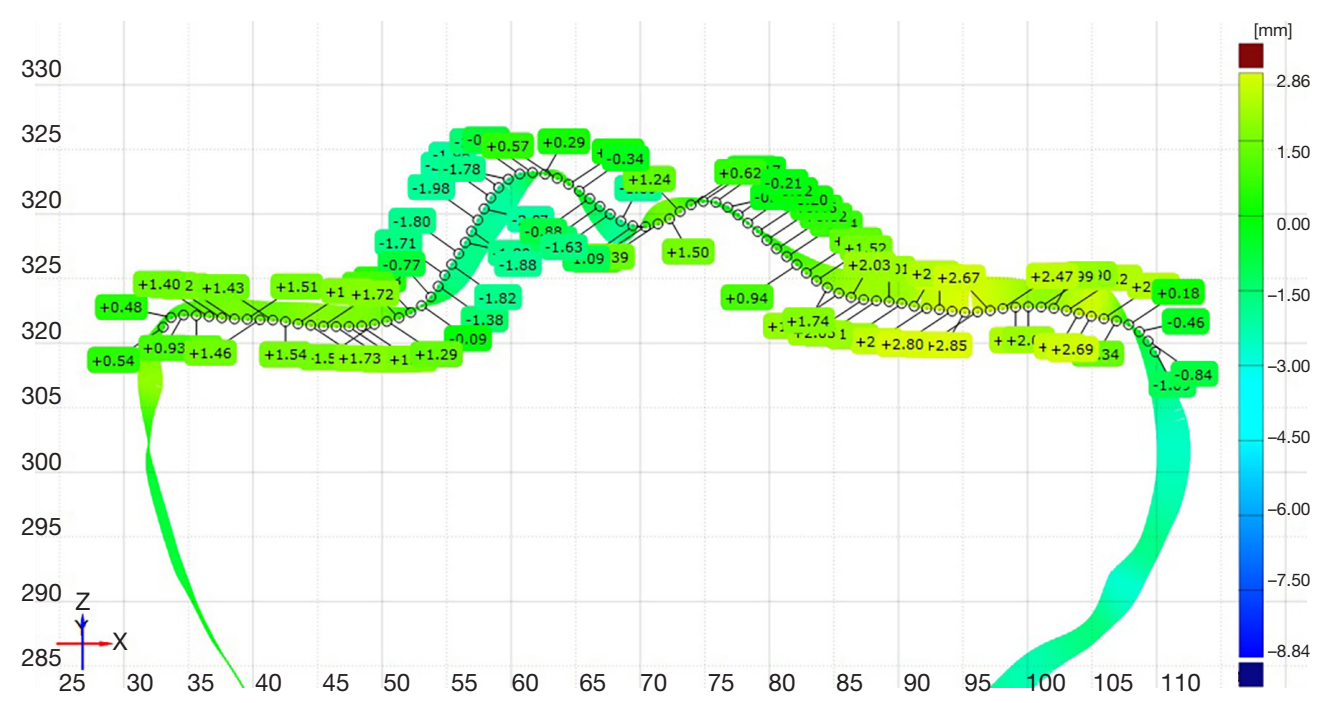

Figure 2 Example of a set of measurements points in 2D cross-section.

\section{Bone width}

First, the bone width was measured in the longest line in the cross-section view at the midplane at the coronal view (Figure 1). The width was calculated separately for each bone using GOM Inspect software.

\section{D dimensions analysis}

$3 \mathrm{D}$ models were compared to the CAD body of most average bone (selected using geometry pre-adjustment). Then cross-section plane was created in the measured center of base bone in the Y-axis view. Differences in the shape in $\mathrm{Z}$-axis were measured by comparing other bones to the base one at the line of the created crosssection.

Models alignment was automatically calculated by the software, according to the middle line. For every measurement, the result was a set of points at equal distance $(0.50 \mathrm{~mm})$ in the $\mathrm{X}$-axis (Figure 2$)$. All measured points were in the same position according to gender and side of the body (right/left leg).

A colorful map of deviations was created for better visualization (legend on the right side in Figure 2). 


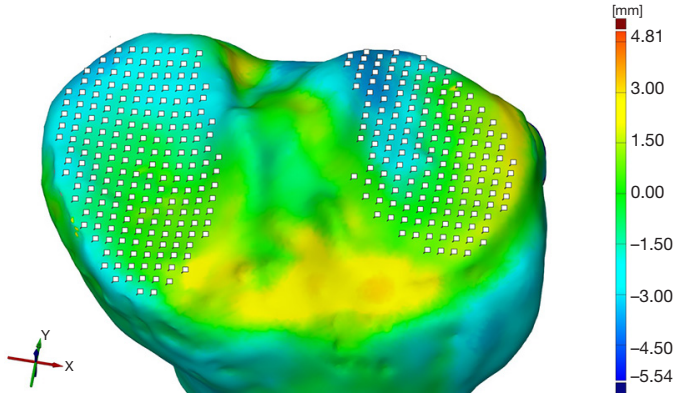

Figure 3 Example of a map of deformation with the cloud of measurements points.

\section{D dimensions analysis}

The differences in dimension over the entire surface of the plateau were measured by generating a deformation map on whole geometry. Again, in this case, all 3D models were compared to the basic one. Then the set of measurement points was generated and calculated separately for the medial and lateral plateau. In this case measurement points were created at an equal distance of $1.20 \mathrm{~mm}$ (Figure 3).

\section{Plateau surface area}

The surface area for each plateau separately was measured using the SpaceClaim module for ANSYS software. The surface area was determined according to the highest points of geometry as compared to the lowest points in the plateau area (Figure 4).

\section{The angle of inclination of the plateau}

To measure the angle of inclination of the plateau at first bones were aligned like in section "2D dimensions analysis" and "3D dimensions analysis". The orientation of the reference plane is based on the center axis of the bone. Two reference planes were added-one in ZY-axis (Figure $5 A$ ) and second-in ZX-axis (Figure $5 B, C$ ). The last step was the measurement of the angle between the reference plane and planes of the plateau. An example of the measured angles is shown in Figure 6. The procedure was the same for every single 3D mesh. Every comparison used the same reference plane.

\section{Statistical analysis}

Data obtained by measurements were tested using a single factor ANOVA test statistical significance assumed at a level of $\mathrm{P}<0.05$. Statistical analysis was the determination of mean and median and standard deviation (SD). The fille

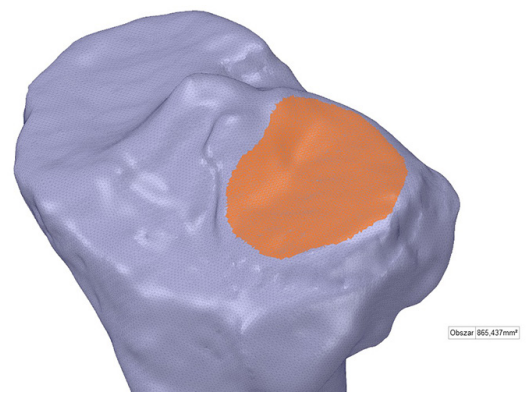

Figure 4 Example of measurement of plateau surface area.

area limits are the minimum and maximum values.

\section{Results}

\section{Bone width}

The male tibial plateau was much larger than the female one. There was a significant difference between genders for the right $(\mathrm{P}=0.001)$ and left knees $(\mathrm{P}=0.001)$. An average measured width for the females was: $73.60 \pm 3.83 \mathrm{~mm}$ (range, 68-81) for left knees and 73.10 $\pm 3.23 \mathrm{~mm}$ (range, 67.5-79.4) for right knees, and this difference was not statistically significant $(\mathrm{P}=0.62)$. A mean width value for males was $83.28 \pm 4.01 \mathrm{~mm}$ (range, 74.8-92.6) for left knees and $82.32 \pm 3.62 \mathrm{~mm}$ (range, 74.7-91.7) for right knees, and this difference was not statistically significant. Results of all our calculations are presented in Table 1.

Measured average bone width is shown in Figure 7.

\section{$2 D$ dimensions analysis}

Graphical illustrations of calculated 2D deviations at crosssection analysis are shown in Figure 8. Male knees showed much bigger variability in tibial plateau dimensions then female ones but these differences were significant only for the left knees $(\mathrm{P}=0.001)$. There was also a marked difference in 2D dimension variability between sides in males (Table 1).

\section{$3 D$ dimensions analysis}

Variability in 3D dimensions was statistically significant for medial condyles between sides for both genders, as right knees showed greater changes for both sexes (Table 1). Inside the male population, this difference was also significant for lateral condyles. Between sexes, there was a much bigger 


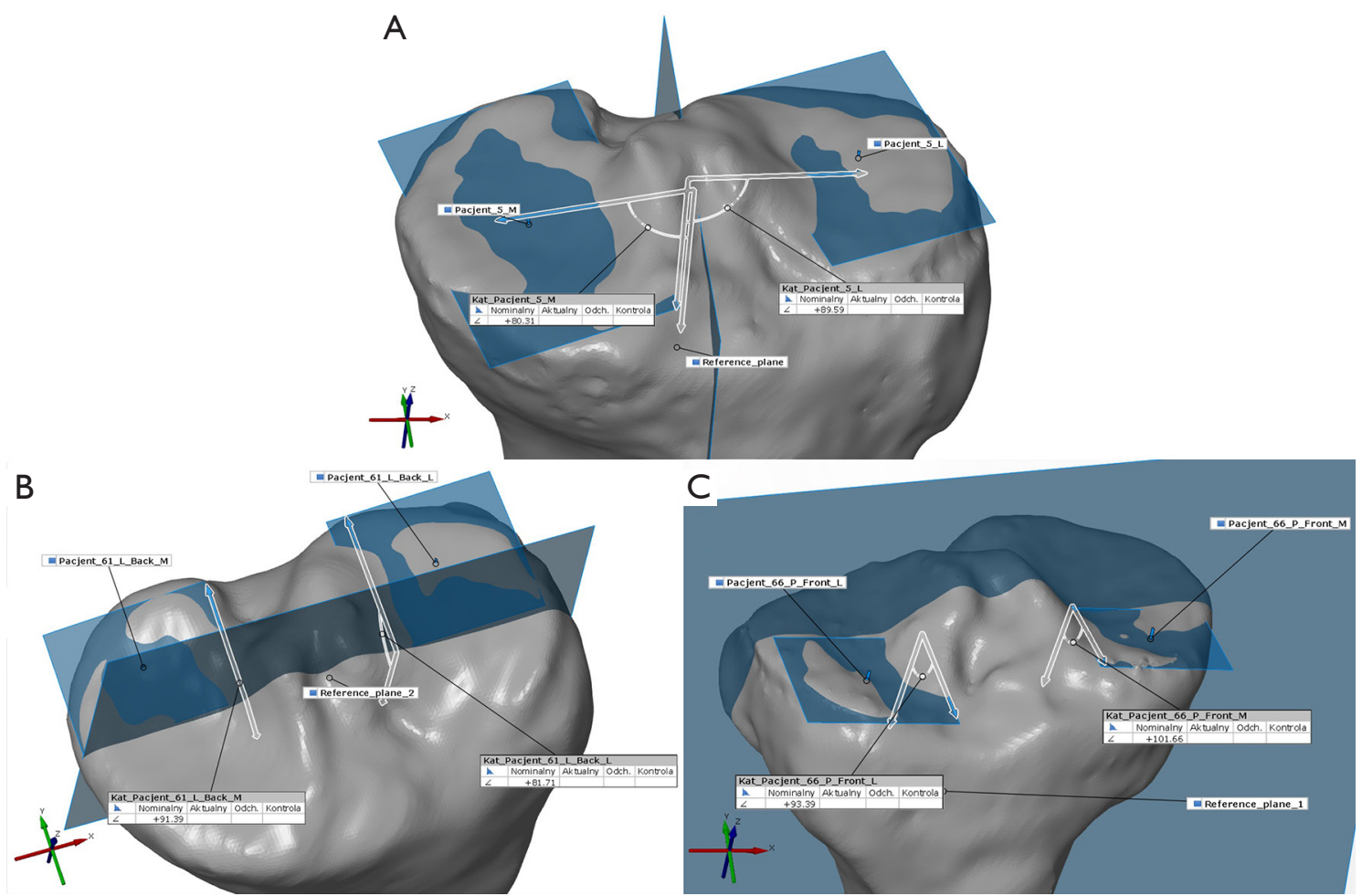

Figure 5 Example of measurement of inclination of the plateau: (A) sides, (B) front, (C) back.

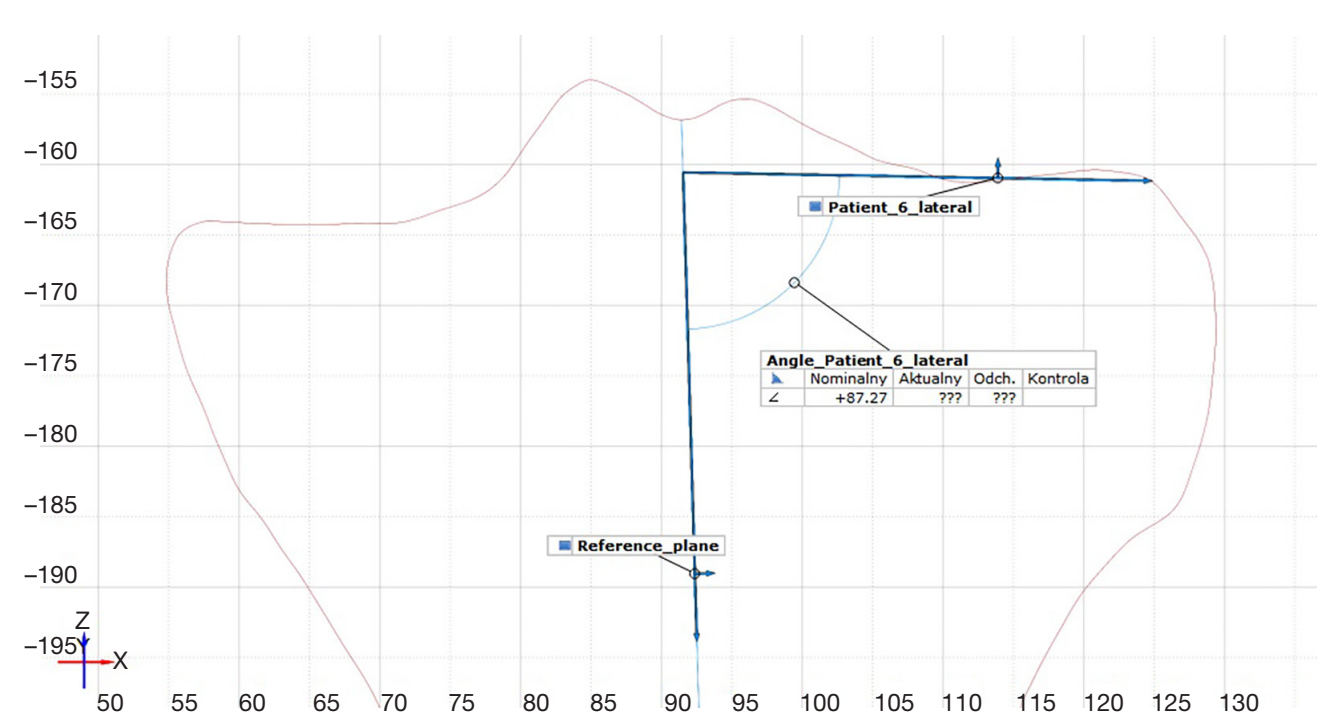

Figure 6 Example of measurement of inclination of plateau.

variability $(\mathrm{P}=0.001)$ in male knees for both sides and both condyles. In Figure 9 are shown diagram results for each gender. Results of the created map of deviations show average differences in the whole area of the plateau (Figure 10).

\section{Plateau surface area}

There were no significant differences in the measured surface area of each plateau between sides for either condyle (Table 1). Male knees had a statistically bigger $(\mathrm{P}=0.04)$ 
Table 1 Results of the calculations

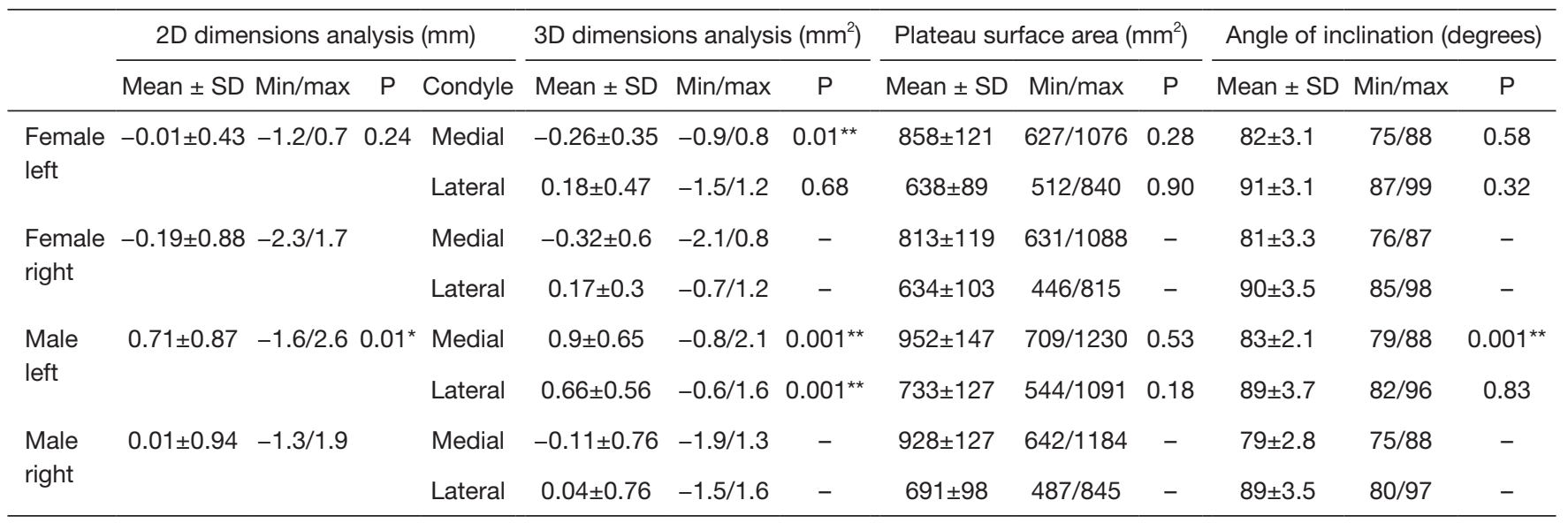

$P$ value of Manna-Whitney $U$ test, significance $P<0.05$ marked with * when tested between sides within same gender; marked ${ }^{* *}$ when tested between single-name condyles within same gender. N, number of cases; SD, standard deviation; min, minimum; max, maximum.

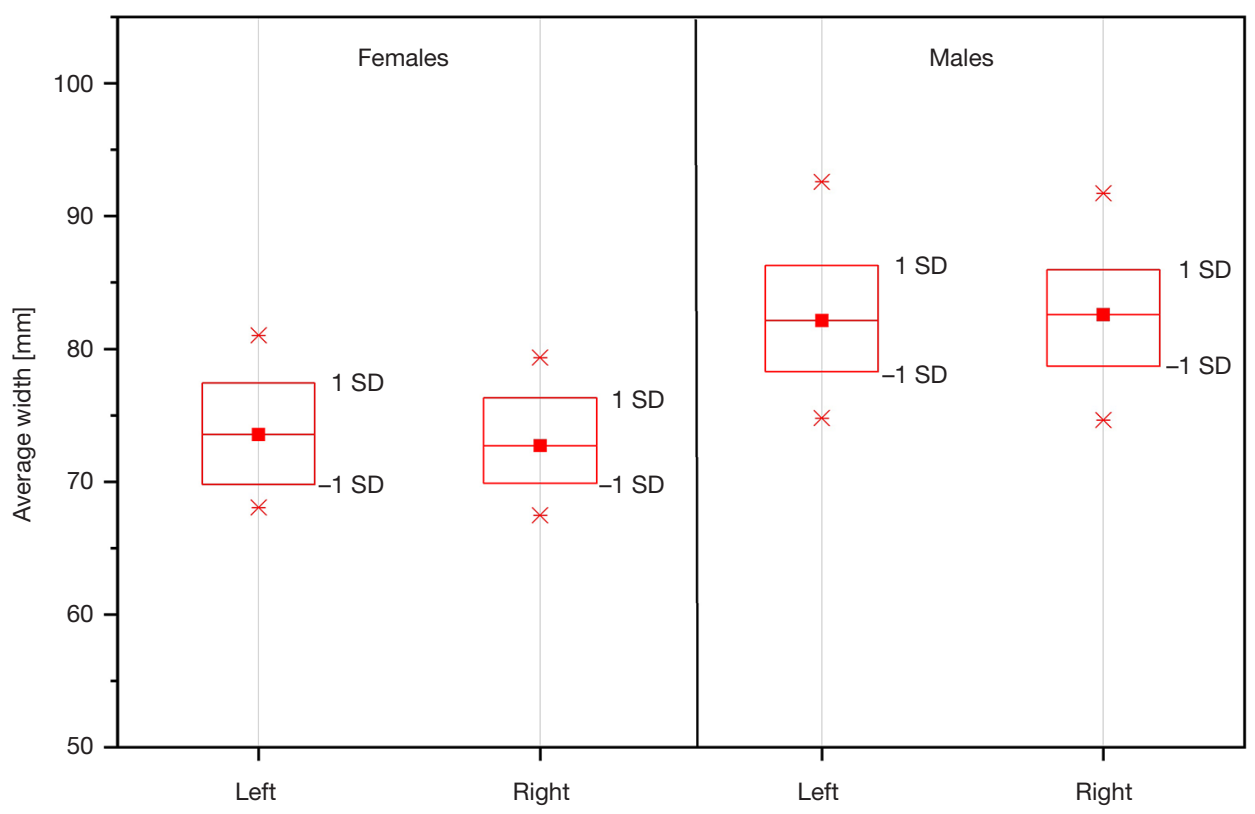

Figure 7 Average bone width.

surface area for all measured condyles.

The final results of measurement of the surface area of each plateau are shown in Figure 11.

\section{The angle of inclination of the platean}

Angles of inclination of the medial condyle plateau differ statistically in the male population (Table 1). A significant difference between sexes was observed only for medial condyles of the right knees $(\mathrm{P}=0.02)$, as males had lower angle values.

Results of calculated average angles of inclination of a plateau concerning the central axis are shown in Figure 12.

\section{Discussion}

The contralateral bone is often used as a reference field in clinical practice. This approach assumes that there are 

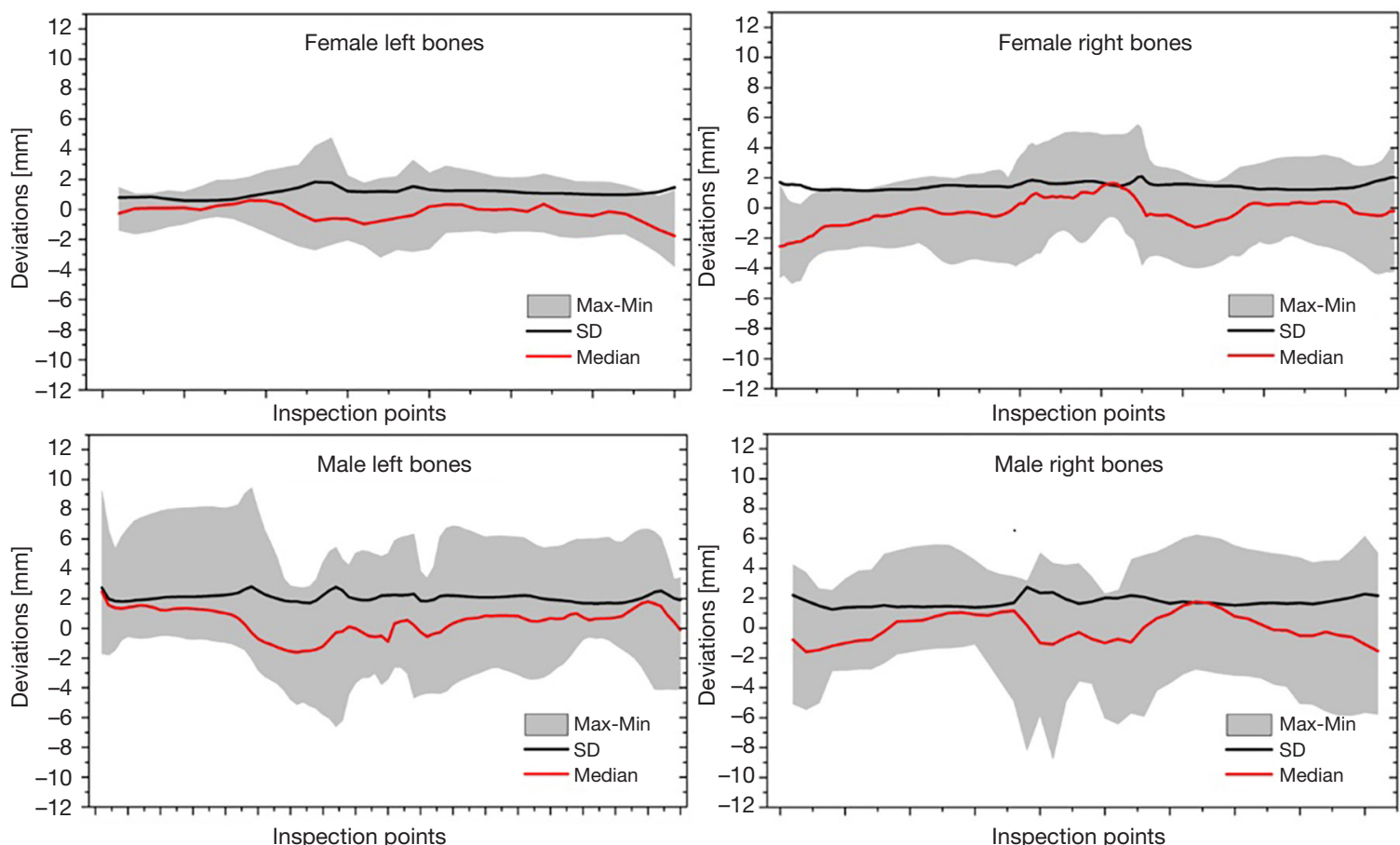

Figure 8 Graphical illustrations of 2D deviations of the tibial plateau.

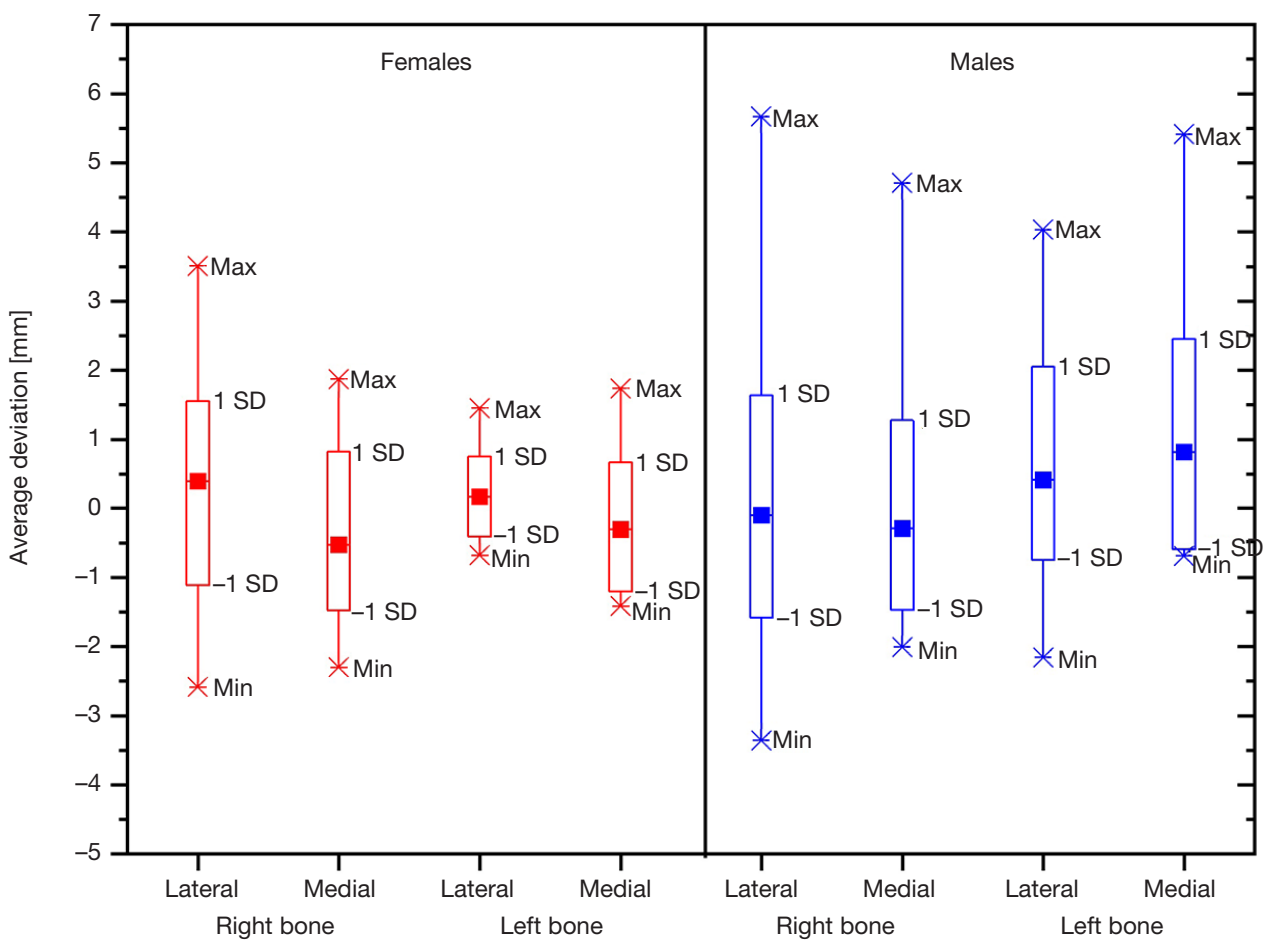

Figure 9 The average dimensional difference for each plateau for each gender. 

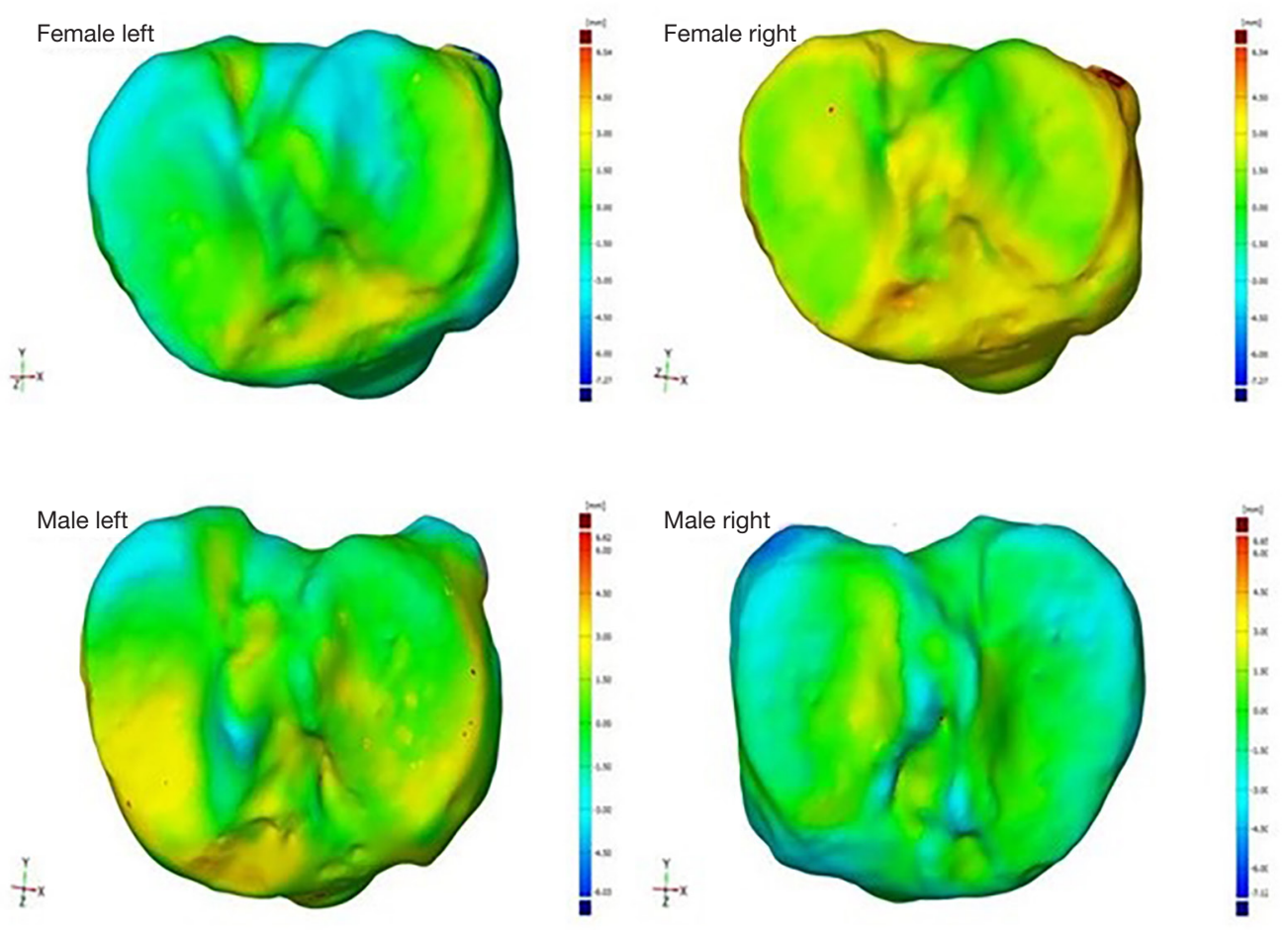

Figure 10 Graphical illustrations of 3D deviations of the tibial plateau.

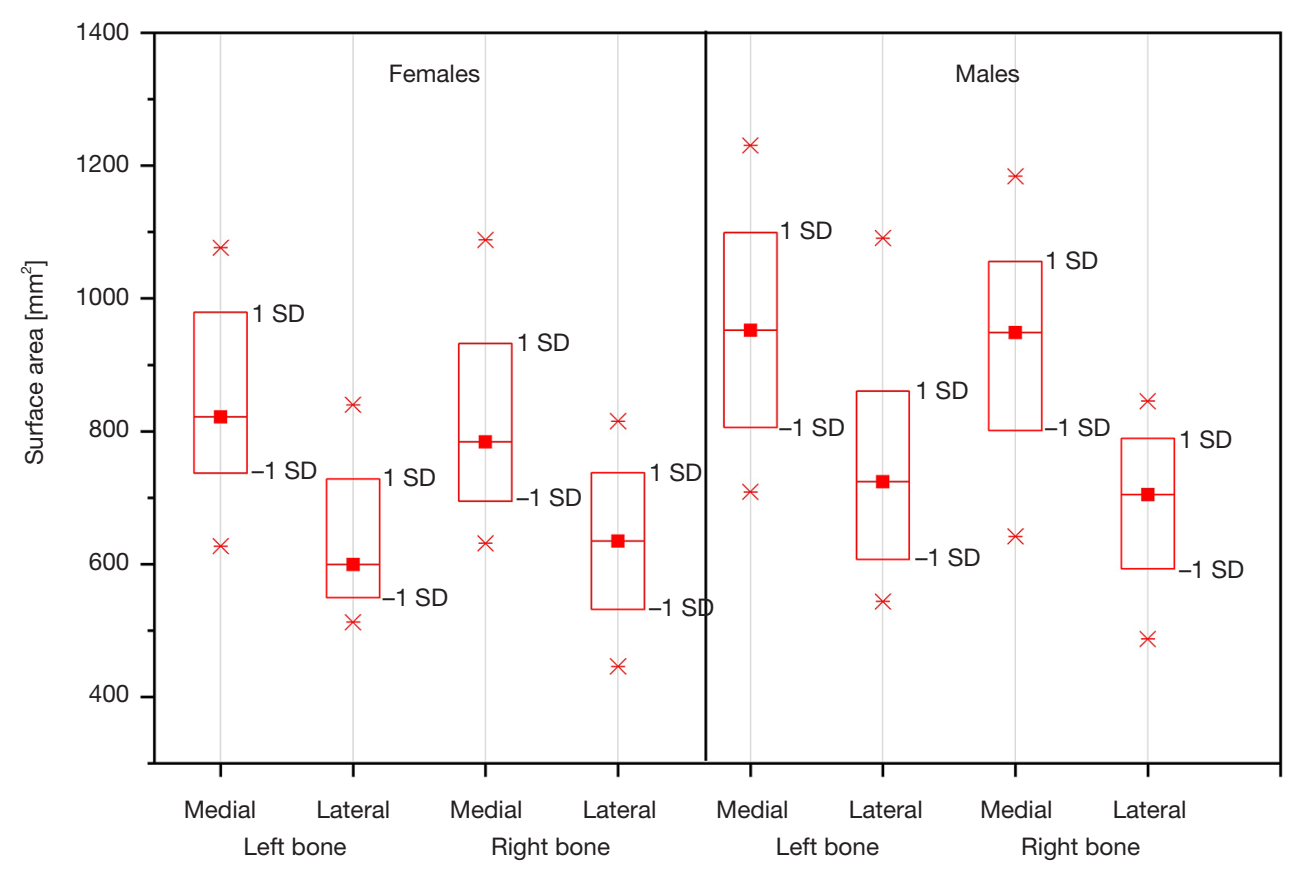

Figure 11 Results of measurements of the average surface area of each plateau. 


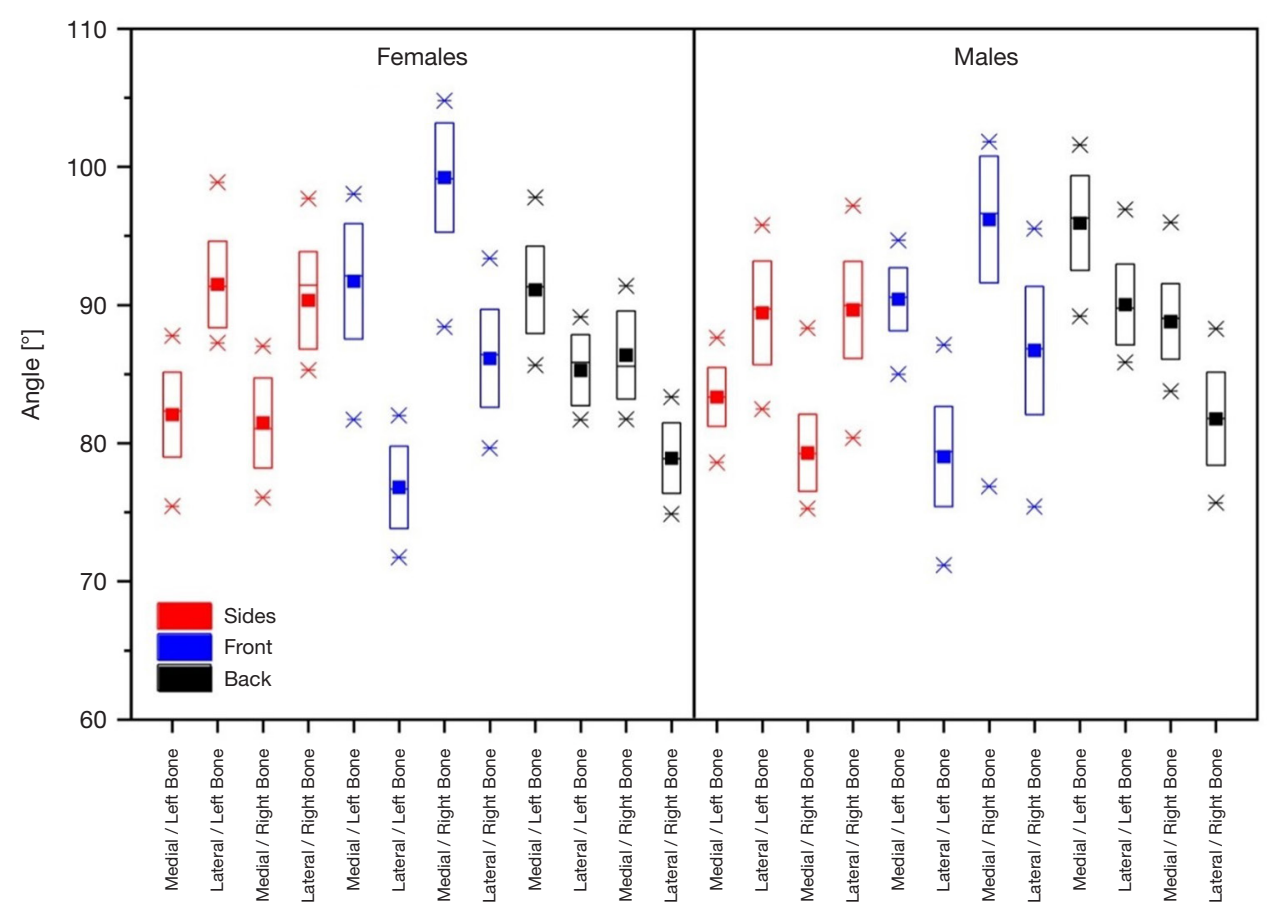

Figure 12 Diagram of calculated angles of inclination of the plateau.

no significant differences in bones according to side of the body, even though previous studies have reported bilateral asymmetries in long bones $(8,9)$. Defining anatomical variations of the tibia is of importance not just for surgeons, but also for researchers and commercial partners.

In this study we aimed to evaluate anatomical variations of proximal tibia plateau, by creating and analyzing threedimensional mathematical model. The male knees were much larger, and showed higher variabilities in dimensions especially for the left knees and medial condyles. Described differences may be even bigger in osteoarthritic knees and it will be analyzed in the future basing on the described model.

Many previous research known to us $(10,11)$ were based on cadavers study. The above works concerned information about the whole length of the bone. The biggest difference was noted in the length of the bone according to the gender. Our control group was constituted of CT scans of the knees. In our study parameter adequate to the length, connected with the size of the bone, is epiphysis width and plateau surface area which were most variable according to gender. There was no statistically significant difference in our study in plateau surface area according to the side of the body, what is interesting for the sake of many of our specimens came from different patients (in many cases in posttraumatic patients one of the bones was excluded).

There are some anthropological studies, which have reported that the left bone is larger than right in lower limb, though there is less bilateral variations in the lower compared to the upper limb (8). Plochocki in his survey proved directional asymmetry, which is probably due to differential mechanical loading during bone growth related to handedness. Further research is needed to establish the exact relationship between joint size and the mechanical environment.

There are also some studies, wich confirm that bone shape of the tibia and femur differ by sex and the knee bone shape is associated with incident knee radiographic OA (12). What is more they observed that some distal femur and proximal tibia shape appear to protect women from developing OA.

In 2D dimensions analysis, we obtained greater variability in measurements in men group with statistically significant importance for the left side of the body. Ingelmark (13) observed that a right-handed person is likely to have a more developed contralateral left lower limb due to an increase in its muscle in order to compensate and support the right upper limb. And as Peters et al. (14) reported more than 
$77 \%$ of population shows a right hand preference. That made us thinking that above differences may come from dominant and non-dominant limbs variations.

In $3 \mathrm{D}$ dimensions' analysis of models, there was statistically significant importance in the area of medial condyle for both genders and sides, wherein right-sided bones in both sexes revealed greater variation.

In men's population statistically, significant differences in the area of lateral condyle were observed.

Quintens et al. (11) reported statistically significant difference in mid diaphyseal region and medial tibia plateau, but the limitation of their study was no division according to sex or age.

In our study holistically the epiphyses of the tibia in the group of men in 3D model analysis revealed greater volatility compares to the group of women.

Angles of inclination of medial condyle plateau differ statistically in the male population. A significant difference between sexes was observed only for medial condyles of the right knees males had lower angle values. As $\mathrm{Ng}$ et al. proved there is also correlation between proximal tibial joint line obliquity and distal femoral rotational geometrythe more varus the tibial plateau inclination is in the coronal plane, the more internally rotated distal femur is (15).

Advantages of our study is division according to gender and sides of the body in group of patients between 20-40 years old. From hospital database CT scans of healthy knees were collected and we studied both, 2D and $3 \mathrm{D}$ measurements. Besides being able to quantify the surface deviations, the use of $3 \mathrm{D}$ models allow a better understanding a detailed representation and accuracy of the complexity of their anatomical bony structure.

Limitation of the study, without a doubt, is a field of the research given the fact that we were analyzing proximal tibia epiphysis, so we couldn't correlate results with the rest part of the bone. It would be very interesting to correlate our results with height or weight, but as we used clinical database and CT scans of patients mostly form emergency room, we could not obtain this data. However as Sharma et al. proved body mass index (BMI) is related to severity of OA in varus alignment but not in valgus alignment (16) and it is dependent on specifics of malalignment in the knee, which differ by sex.

Our study showed that parameters that result from the size of the bone are not the only variable value. We suppose that differences according to the side of the body may be the result of dominant and non-dominant limb, but to prove it further research is needed.
Finally, the size of the bone is not the only differential parameter according to gender. 2D and 3D dimension analysis of the plateau area is more variable in the male group.

The above data shed some light on orthopedic operation planning.

\section{Conclusions}

In conclusion, the accurate quantification of bilateral asymmetries of the tibia requires both $2 \mathrm{D}$ and $3 \mathrm{D}$ measurements. We believe that this large anatomical variability of the tibia needs to be taken into consideration during preoperative planning to avoid complications such as intraoperative fractures or inadequate reductions and/ or fixations, which in result lead to biomechanical and functional problems.

We suspect that those asymmetries may be even more pronounced in OA knees and complications such as lateral hinge fracture may be an effect of overloading the lateral compartment. We believe second part of our study will bring useful information in this regard.

\section{Acknowledgments}

Funding: None.

\section{Footnote}

Conflicts of Interest: All authors have completed the ICMJE uniform disclosure form (available at https://dx.doi. org/10.21037/qims-20-1094). The authors have no conflicts of interest to declare.

Ethical Statement: The authors are accountable for all aspects of the work in ensuring that questions related to the accuracy or integrity of any part of the work are appropriately investigated and resolved. The study was conducted in accordance with the Declaration of Helsinki (as revised in 2013). An approval from the Local University Ethical Committee was obtained for the study design and data acquisition from CT scans. Because our study did not disclose any private patient data, individual consent for this retrospective analysis was waived.

Open Access Statement: This is an Open Access article distributed in accordance with the Creative Commons Attribution-NonCommercial-NoDerivs 4.0 International 
License (CC BY-NC-ND 4.0), which permits the noncommercial replication and distribution of the article with the strict proviso that no changes or edits are made and the original work is properly cited (including links to both the formal publication through the relevant DOI and the license). See: https://creativecommons.org/licenses/by-nc-nd/4.0/.

\section{References}

1. Radzi S, Uesugi M, Baird A, Mishra S, Schuetz M, Schmutz B. Assessing the bilateral geometrical differences of the tibia--are they the same? Med Eng Phys 2014;36:1618-25.

2. Dargel J, Ninck J, Koebke J, Appell HJ, Pennig D, Hillekamp J. Influence of knee flexion on plantarflexion moments after open or percutaneous Achilles tendon repair. Foot Ankle Int 2009;30:551-7.

3. Eckhoff DG, Jacofsky DJ, Springer BD, Dunbar M, Cherian JJ, Elmallah RK, Mont MA, Greene KA. Bilateral Symmetrical Comparison of Femoral and Tibial Anatomic Features. J Arthroplasty 2016;31:1083-90.

4. Dobbe JG, Pré KJ, Kloen P, Blankevoort L, Streekstra GJ. Computer-assisted and patient-specific 3-D planning and evaluation of a single-cut rotational osteotomy for complex long-bone deformities. Med Biol Eng Comput 2011;49:1363-70.

5. Santoro D, Tantavisut S, Aloj D, Karam MD. Diaphyseal osteotomy after post-traumatic malalignment. Curr Rev Musculoskelet Med 2014;7:312-22.

6. Schröter S, Freude T, Kopp MM, Konstantinidis L, Döbele S, Stöckle U, van Heerwaarden R. Smoking and unstable hinge fractures cause delayed gap filling irrespective of early weight bearing after open wedge osteotomy. Arthroscopy 2015;31:254-65.

7. Jackson JP, Waugh W, Green JP. High tibial osteotomy for osteoarthritis of the knee. J Bone Joint Surg Br 1969;51:88-94.

8. Plochocki JH. Bilateral variation in limb articular surface dimensions. Am J Hum Biol 2004;16:328-33.

9. Auerbach BM, Ruff CB. Limb bone bilateral asymmetry: variability and commonality among modern humans. J Hum Evol 2006;50:203-18.

10. Schenk P, Vlachopoulos L, Hingsammer A, Fucentese SF, Fürnstahl P. Is the contralateral tibia a reliable template for reconstruction: a three-dimensional anatomy cadaveric study. Knee Surg Sports Traumatol Arthrosc 2018;26:2324-31.

11. Quintens L, Herteleer M, Vancleef S, Carette Y, Duflou J, Nijs S, Sloten JV, Hoekstra H. Anatomical Variation of the Tibia - a Principal Component Analysis. Sci Rep 2019;9:7649.

12. Wise BL, Niu J, Zhang Y, Liu F, Pang J, Lynch JA, Lane NE. Bone shape mediates the relationship between sex and incident knee osteoarthritis. BMC Musculoskelet Disord 2018;19:331.

13. Ingelmark BE. Asymmetries in the length of the extremities and. their relation to right- and lefthandedness. Upsala Lakareforen Forh 1974;52:17-82.

14. Peters M, Reimers S, Manning JT. Hand preference for writing and associations with selected demographic and behavioral variables in 255,100 subjects: the BBC internet study. Brain Cogn 2006;62:177-89.

15. Ng CK, Chen JY, Yeh JZY, Ho JPY, Merican AM, Yeo SJ. Distal Femoral Rotation Correlates With Proximal Tibial Joint Line Obliquity: A Consideration for Kinematic Total Knee Arthroplasty. J Arthroplasty 2018;33:1936-44.

16. Sharma L, Lou C, Cahue S, Dunlop DD. The mechanism of the effect of obesity in knee osteoarthritis: the mediating role of malalignment. Arthritis Rheum 2000;43:568-75.

Cite this article as: Tomczyk J, Taczała J, Sawicki J, Domżalski M. Assessment of morphological differences of the proximal tibia in healthy knees: analysis of the 3-dimensional mathematical model. Quant Imaging Med Surg 2021;11(10):4354-4364. doi: 10.21037/qims-20-1094 